\title{
Advisability of primary intraocular lens implantation for infants under 2: A systematic review and meta-analysis
}

\author{
Shanshan Jin ${ }^{1}$, Jing Shang Zhang ${ }^{2}$, Jin Da Wang${ }^{2}$, Yusufu Mayinuer ${ }^{2}$, Kai Cao ${ }^{1}$, Guyu \\ Zhu ${ }^{1}$, Hailong $\mathrm{He}^{1}$, and Xiu Wan ${ }^{1}$ \\ ${ }^{1}$ Beijing Institute of Ophthalmology \\ ${ }^{2}$ Affiliation not available
}

September 11, 2020

\begin{abstract}
Purposes: The present meta-analysis compared the postoperative visual performance of primary intraocular lens (IOL) implantation and primary aphakia in cataract infants. Methods: We performed a systematic literature search in PubMed, EMBASE and Science Direct. Postoperative visual acuity (VA) and complications were extracted and pooled. Results: Four randomized controlled trails (RCTs) and seven retrospective studies were included. The postoperative VA in primary IOL group was better than that in primary aphakia group $[\mathrm{MD}=-0.12,95 \% \mathrm{CI}:(-0.19,-0.05), \mathrm{p}=0.91]$. There was no significant difference in the incidence of glaucoma, retinal detachment and nystagmus between primary IOL group and primary aphakia group [OR=1.27, $95 \%$ CI: $(0.79,2.05), \mathrm{p}=0.84$ for glaucoma; $\mathrm{OR}=0.49,95 \% \mathrm{CI}:(0.07,3.30), \mathrm{p}=0.34$ for retinal detachment; $\mathrm{OR}=1.11,95 \% \mathrm{CI}$ : $(0.62,1.98), \mathrm{p}=0.73$ for nystagmus]. Analysis of unilateral subgroup indicated there were fewer infants with strabismus in primary IOL group compared with primary aphakia group [OR $=0.40,95 \%$ CI: $(0.21,0.79), \mathrm{p}=0.46]$. The primary IOL group needed more visual axis opacification (VAO) clearing than primary aphakia group [OR=9.33, 95\%CI: $(5.21,16.73)$, p=0.27]. Conclusion: Primary IOL implantation provided more visual benefits. IOL implantation may decrease the incidence of strabismus in comparison with primary aphakia in unilateral subgroup. However, these advantages could be offset by a higher VAO clearing surgery.
\end{abstract}

\section{Extra information}

\section{How did you gather the information you considered in your review?}

We performed a systematic search in PubMed, Science Direct, EMBASE and Cochran Library. One or a combination terms was used in the search. The publication time of the literature was restricted from January 1, 2000 to August 2, 2020. Two investigators searched and screened the articles independently. In case of disagreement about the literature search, a third reviewer would engage in the discussion until a consensus was reached.

\section{What is the 'take-home' message for the clinician?}

We found the postoperative VA of primary IOL group was better than that of primary aphakia group. Actually, constant optical correction during this crucial period is very important for children. Moreover, previous studies indicated primary IOL implantation was a protective factor of secondary glaucoma. Whereas we found there was no difference in the incidence of glaucoma surgery between this two groups. These two critical findings help the surgeons to weigh the visual benefits and the risks of surgery when they choose IOL implantation for a children younger than 2 years.

Keywords : Primary intraocular lenses, Primary aphakia, Cataract surgery, Cataract infant; 


\section{Introduction}

Approximate 1.4 million children suffer from blindness around the world. ${ }^{1}$ Childhood preventable blindness has been identified as a priority of the Vision 2020: "The Right to Sight" ${ }^{2}$. Pediatric cataract is the leading cause of treatable childhood blindness and accounts for $5-20 \%$ of childhood blindness ${ }^{3-5}$. IOL implantation is the main option for optical rehabilitation in children after cataract surgery. There are two main surgical procedures for IOL implantation in cataract infants, i.e. primary IOL implantation and primary aphakia followed by secondary IOL implantation. Due to the risk of postoperative complications, such as inflammatory responses, VAO and secondary glaucoma, IOL implantation in cataract children older than 2 years has been a consensus among pediatric cataract surgeons around the world ${ }^{6-9}$. But in other cases, children with aphakic spectacles or contact lenses are susceptible to VA issues, corneal problems, compliance with lens-wearing and high cost.

Many studies had reported the clinical performance (including VA and postoperative complications) after congenital cataract surgery ${ }^{7,10-12}$. However, given the elongation of axial length, change of corneal curvature and high incidence of additional surgery, the benefits and risks of primary IOL implantation in cataract infants, especially in those younger than 2 years, still remains a controversial issue ${ }^{13-15}$. Surgeons need to weigh the visual benefits agains risk (such as inflammatory response, VAO and secondary glaucoma associated with the procedure) of surgery when they choose IOL implantation for a children younger than 2 years.

Glaucoma is the main complication of congenital cataract surgery and has been discussed in many studies $^{16-18}$. However, there is no consistent conclusion on which type of surgery can decrease the incidence of glaucoma. Some studies suggested that primary IOL implantation was a protective factor of glaucoma after cataract surgery ${ }^{19,20}$. Whereas, other studies suggested that the incidences of glaucoma were comparable in primary IOL group and primary aphakia group ${ }^{11,21,22}$. Similarly, previous studies didn't reach consensus on which type of procedure would be better in terms of postoperative VA and complications ${ }^{23,24}$. Therefore, the present meta-analysis aims to compare the postoperative performance of primary IOL implantation and primary aphakia in cataract infants younger than 2 years, and provide solid evidence for better clinical practice.

\section{Materials and methods}

\section{Search strategy}

A systematic search was performed in PubMed, Science Direct, EMBASE and Cochran Library. One or a combination of the following terms was used in the search: congenital, infant, child, pediatric, cataract, intraocular lens, IOL, primary, secondary, pseudophakia, aphakia, cataract surgery, comparison and compare. The publication time was restricted from January 1, 2000 to August 2, 2020. Two investigators (Shanshan Jin and Jingshang Zhang) searched and screened the articles independently. In case of disagreement about the literature search, a third reviewer (Xiuhua Wan) would engage in the discussion until a consensus was reached. Figure 1 showed the selection process.

\section{Inclusion and exclusion criteria}

Inclusion and exclusion criteria: (i) The subjects should be cataract infants younger than 2 years $(\mathrm{P})$ who received cataract extraction with primary IOL implantation (I) or primary aphakia (C); (ii) The clinical outcomes on postoperative VA, complications (glaucoma, strabismus, nystagmus and visual axial opacity) (O) should be evaluated; (iii) Given the age at the surgery and the age at the last visit were significant factors for the postoperative outcomes in congenital cataract patients, studies in which the age at the surgery or last visit in these two groups did not match were excluded; (iv) Studies without detailed outcome on the postoperative visual performance were excluded; (v) Studies reporting data on individual patient rather than individual surgery eye were excluded; (vi) (potentially) overlapping study populations that reported same outcome were excluded; (vii) Non-English publications were excluded.

\section{Data extraction}


Data were extracted using pre-defined data fields from each study by two investigators independently. If there were inconsistencies between two investigators, a third reviewer would extract results and engage in discussion until a consensus was reached. VA reported as log MAR VA were extracted as the primary outcome. Second outcomes including glaucoma, strabismus, nystagmus and VAO were extracted. The results of postoperative visual performance at last visit were extracted from studies with multiple postoperative follow-up data. As for the retrospective studies, age-matched outcome would be extracted if reported. Continuous data reported as mean \pm SD were extracted directly. Those data reported as median and interquartile ranges would be converted into mean and SD based on the formula ${ }^{25}$.

\section{Quality assessment}

The quality of the included RCTs was assessed with the Cochrane Risk of Bias Tool ${ }^{26}$. It contained six domains: randomization process, intervention deviation, missing outcome data, outcome measurement, selective outcome reporting. The retrospective studies were assessed by the Newcastle-Ottawa Scale (NOS) in the following 3 aspects: cohort selection, cohort comparability, assessment of outcome ${ }^{27}$. Details of NOS were available in Appendix 1.

\section{Data synthesis and statistical analysis}

The statistical analysis was performed with the statistical software open source $\mathrm{R}$ program (Version 3.41). Categorical outcomes were estimated by odds ratio (OR) with 95\% CI. If "1" falls into the CI, the outcome would be considered "not statistically significant". The mean difference (MD = mean of primary IOL - mean of primary aphakia) with a $95 \%$ confidence interval (CI) was adopted for continuous outcomes. If "0" falls into the CI, the outcome would be considered "not statistically significant".

Heterogeneity across studies was tested with $\mathrm{Q}$ test and $\mathrm{I}^{2}$ statistic. If there was no heterogeneity across these studies ( $\mathrm{I}^{2} ; 50 \%$ ), the Mantel-Haenszel fixed-effect model would be used. $\mathrm{I}^{2}$ above $50 \%$ and the P-value below 0.1 would constitute a significant heterogeneity among these studies, and possible reasons would be explored by reviewing the included studies. Three approaches were used to detect the source of heterogeneity in this meta-analysis: sensitive analysis, subgroup analysis and meta regression. Through sensitivity analysis, we could determine whether the heterogeneity would decrease following exclusion of each study one by one. Subgroup analysis and meta regression would be performed according to the clinical characteristics of the included studies. Through these two approaches, we could identify the factor that induced heterogeneity in the meta-analysis. If sensitivity analysis and subgroup analysis did not decrease the heterogeneity, the random-effects model (DerSimonian-Laird) would be adopted to calculate pooled effect size. ${ }^{28,29}$.

\section{Results}

\section{Characteristics of included studies}

Four randomized controlled trails (RCTs) and seven retrospective studies were included. Table 1 describes the characteristics of the 11 included studies that were published during 2001-2018. The surgery age of subjects ranged from 1.8-7.5 mouth. The follow-up duration ranged from 0.5-5 years.

\section{Risk of bias assessment}

Three RCT studies reported the randomization process, intervention deviation, yet none of them had selective reporting and outcome data missing. The study of Qian $\mathrm{L}^{30}$ did not describe the approach of random sequence generation and potential intervention deviation. Vasavada et al. ${ }^{22}$ reported that the surgery type was revealed to the surgeon and outcome examiner in their study. The details of risk assessment for these RCTs were presented in the Figure 2.

Retrospective studies were assessed based on NOS. Five studies described the detailed process of cohort selection, and were assessed to be of low risk in cohort selection. The study by Autrata et al. ${ }^{31}$ did not report the source of study subjects, and was assessed to be of medium risk in cohort selection. High risk was found in the cohort selection in the study by Trivedi et $\mathrm{al}^{32}$, as the source and preoperative data on patients were not described. Given the age at the surgery and the age at the last visit were significant factors for the 
postoperative outcomes in cataract infants, these two factors were used to assess the cohort comparability. According to our inclusion and exclusion criteria, all these retrospective studies have similar age at surgery and last visit in both groups. So, all these studies were assessed to be of low risk in the cohort comparability. The study by Trivedi et $\mathrm{al}^{32}$. was assessed to be of high risk since they did not report the duration of follow up and rate of follow-up loss. Except for the above study, all the other studies gave detailed information on

outcome assessment. The details of risk assessment for these retrospective studies were shown in the Figure 3.

\section{Visual acuity}

There were 7 studies reporting VA ${ }^{11,22,30,31,33-35}$, and the VA of primary IOL group was similar to that of primary aphakia group $[\mathrm{MD}=-0.12,95 \% \mathrm{CI}:(-0.19,-0.05), \mathrm{p}=0.91]$. There was no heterogeneity $\left(\mathrm{I}^{2}\right.$ $=0 \%)($ Figure 4$)$. No publication bias was found (Table 2).

\section{Glaucoma}

Glaucoma was reported in 7 studies $^{11,22,30,32,34,36,37}$, there was no heterogeneity $\left(\mathrm{I}^{2}=0 \%\right)$. Therefore, the fixed effect model was applied. As shown in Figure 5, there was no difference in the incidence of glaucoma between primary IOL group and primary aphakia group [OR=1.27,95\% CI: $(0.79,2.05), \mathrm{p}=0.84]$.

\section{Strabismus}

As Figure 6 shows, 6 studies reported the data on strabismus ${ }^{11,22,31,33-35}$. There were much fewer infants with strabismus in primary IOL group compared with primary aphakia group after the surgery $[\mathrm{OR}=0.53,95 \% \mathrm{CI}$ : $(0.31,0.90), \mathrm{p}=0.19]$. Given the unilateral cases or bilateral cases will be a critical factor that contribute to strabismus incidence after congenital cataract surgery, a subgroup analysis was performed. There were much fewer infants with strabismus in primary IOL group compared with primary aphakia group in the unilateral subgroup $[\mathrm{OR}=0.40,95 \% \mathrm{CI}:(0.21,0.79), \mathrm{p}=0.46]$. No difference was found in the bilateral subgroup.

\section{Nystagmus}

Four studies reported the outcome on nystagmus incidence ${ }^{22,34,35,38}$, and no heterogeneity was found $\left(\mathrm{I}^{2}=\right.$ $0 \%$ ). No statistically difference was found between the primary IOL and primary aphakia group in terms of nystagmus $[\mathrm{OR}=1.11,95 \% \mathrm{CI}$ : $(0.62,1.98), \mathrm{p}=0.73]$ (Figure 7 ).

\section{Retinal detachment}

As shown in Figure 8, only 2 studies reported data on $\mathrm{RD}^{11,34}$, and there was no statistical heterogeneity among these studies $\left(\mathrm{I}^{2}=0 \%\right)$. No statistically significant difference was found between the two groups $[\mathrm{OR}=0.49,95 \% \mathrm{CI}:(0.07,3.30), \mathrm{p}=0.34]$.

\section{Visual axial opacity clearing}

VAO clearing was reported in 5 studies ${ }^{11,22,34,37}$, and the $\mathrm{I}^{2}$ was $23 \%$. As shown in Figure 9 , the primary IOL had a significantly higher incidence of VAO clearing compared with primary aphakia, $[\mathrm{OR}=9.33,95 \% \mathrm{CI}$ : $(5.21,16.73), \mathrm{p}=0.27]$.

\section{Discussion}

The present meta-analysis demonstrated that the postoperative VA in primary IOL group was better than that in primary aphakia group. These results were consistent with previous studies. Birch et al showed that IOL may support better VA development when a cataract was extracted after age 1, while the difference would taper off with the age reaching 4 years ${ }^{39,40}$. Vasavada et $\mathrm{al}^{22}$. reported that more infants in primary IOL group had documentable VA than aphakia group during early postoperative follow-up, and this trend continued until age 5. This result indicated that the visual rehabilitation was faster in pseudophakia group, especially in early postoperative follow-up. However, in the IATS study, the VA was similar in IOL group and contact lens group both in the first $(1 \text { year old })^{6}$ and last follow-up $(4.5 \text { years old })^{11}$. This could be explained by better compliance of contact lenses or spectacles in the aphakia group. The IATS study offered 
free contact lenses and spectacles for the enrolled subjects. Moreover, trained personnel assessed the vision and monitored the compliance through regular home visits. 41 This may not be carried out in the real world, particularly in developing countries. Actually, constant optical correction during this crucial period is very important for children. A faster visual rehabilitation will impact the activity and overall functional development of the children. Therefore, primary IOL implantation may provide better VA for pediatric patients, especially those under age 5 .

Our meta-analysis did not see any difference in glaucoma incidence. Many hypotheses proposed the mechanisms of glaucoma after infantile cataract surgery. However, there is no unanimous conclusion. The mechanisms of secondary glaucoma of pseudophakia eyes and aphakic eyes might be different. The filtration angle of infants is susceptible to postoperative inflammation. Thus, IOL implantation in infants will cause chronic inflammations that induce changes of iris root or trabecular meshwork (TM $)^{42-44}$. This might explain the glaucoma in pseudophakia eyes. As for aphakic glaucoma, since the mechanical absence of lens in the eye, TM cells would be exposed to the lens epithelial cells (LEC). Michael et al. specified that LEC induced changes in TM cells that resembled changes in primary open-angle glaucoma ${ }^{45,46}$. It is worth noting that the mechanism of glaucoma is still unclear. But we could not make any attempt to investigate the mechanisms of secondary glaucoma after infantile cataract surgery in this meta-analysis.

Analysis of unilateral subgroup indicated there were much fewer infants with strabismus in primary IOL group compared with primary aphakia group. This was consistent with the studies by Autrata et al ${ }^{31}$. and Lambert et al. Previous studies also suggested that the incidence of strabismus in IOL group was relatively low ${ }^{47-49}$. The absence of natural lens in unilateral eye will lead to anisometropia and aniseikonia, which are related to secondary strabismus following congenital cataract surgery. Primary IOL implantation offers a stable retinal image with minimal aniseikonia as well as full-time optical correction for surgery eye. Moreover, children have to wear contact lenses or spectacles to get optical correction in case of absent lenses. The compliance of wearing contact lenses will be affected by the complications associated with contact lenses like corneal infection. Hence, this might explain the higher incidence of strabismus in primary aphakia group.

Postoperative VAO is very common in infants after congenital cataract surgery. According to the previously published literatures, IOL implantation is associated with higher VAO incidence in congenital cataract children, especially those younger than 6 months ${ }^{50-52}$. The average incidence of VAO after IOL implantation is $44.0 \%$, while the incidence is up to $80 \%$ when the patient is younger than 6 mouths $^{53}$. The IOL in capsular bag acts as an obstacle and prevents the anterior and posterior capsule leaflets from fusing, while the capsule edges will seal more effectively in aphakic eye. In our meta-analysis, primary IOL group had a noticeably higher incidence of VAO clearing than primary aphakic group, which was consistent with the RCT study conducted by IATS and other previous studies. ${ }^{11,34,54,55}$.

Our meta-analysis has some limitations. First, even though this meta-analysis is the first systematic review on this topic, it included retrospective studies rather than just RCTs. Thus, the level of evidence will be weakened by the inclusion of retrospective studies. However, there is no denying that it is very difficult to conduct RCT among paediatric cataract children. Second, the pooled effect of complication, especially the glaucoma incidence, might be affected by the inconsistent diagnostic criteria adopted in these studies. Third, the control group of these included studies was either primary aphakia followed by contact lens or primary aphakia followed secondary IOL implantation. Third, given the number of patients in each study is relatively small, we could not draw explicit conclusion on the difference in postoperative visual performance.

\section{Conclusions}

In summary, the postoperative VA in primary IOL group was better than that in primary aphakia group. Primary IOL implantation may be of lower incidence of strabismus than those with primary aphakia. However, these advantages could be offset by higher surgery incidence especially VAO clearing.

\section{Contributorship statement}

We thank for support by the sources of funding in this study. Study design and concept of this meta- 
analysis were carried out by Xiu Hua Wan, Jingshang Zhang, Jinda Wang and Shanshan Jin; Shanshan Jin and Jingshang Zhang conducted the data collection; Shanshan Jin and Kai Cao performed the data analysis; Ying Xiong, Guyu Zhu, Jing Li and Hailong He provided interpretation of the data. Shanshan Jin performed the first draft of the manuscript; Shanshan Jin, Jingshang Zhang, Jinda Wang and Xiu Hua Wan revised the manuscript and produced the final version; English polishing was done by Mayinuer Yusufu.

\section{Competing interests}

There is no conflict of interest

\section{Funding}

Beijing new star of science and technology (H020821380190, Z131102000413025), Fund of work committee for women and children of China State department (2014108), National natural science fund (30471861), Beijing Institute of Ophthalmology leading program (201515).

\section{Acknowledgements}

During the preparation of this systematic review, Prof. Ningli Wang and Prof. Ailian Hu of Beijing Institute of Ophthalmology authorized the authors to access the database and put together the whole team to work on this manuscript.

\section{References}

1. Rahi JS, Gilbert CE, Foster A, Minassian D. Measuring the burden of childhood blindness. The British journal of ophthalmology.1999;83(4):387-388.

2. Murray McGavin DD. Global initiative for the elimination of avoidable blindness. Community eye health. $1998 ; 11(25): 3$.

3. Maida JM, Mathers K, Alley CL. Pediatric ophthalmology in the developing world. Current opinion in ophthalmology.2008;19(5):403-408.

4. Adhikari S, Shrestha MK, Adhikari K, Maharjan N, Shrestha UD. Causes of visual impairment and blindness in children in three ecological regions of Nepal: Nepal Pediatric Ocular Diseases Study. Clinical ophthalmology (Auckland, NZ). 2015;9:1543-1547.

5. Khandekar R, Kishore H, Mansu RM, Awan H. The status of childhood blindness and functional low vision in the Eastern Mediterranean region in 2012. Middle East African journal of ophthalmology.2014;21(4):336343.

6. Lambert SR, Buckley EG, Drews-Botsch C, Dubois L, Ward M. A Randomized Clinical Trial Comparing Contact Lens With Intraocular Lens Correction of Monocular Aphakia During Infancy: Grating Acuity and Adverse Events at Age 1 Year. Archives of Ophthalmology.2010;128(7):810-818.

7. Plager DA, Yang S, Neely D, Sprunger D, Sondhi N. Complications in the first year following cataract surgery with and without IOL in infants and older children. Journal of AAPOS : the official publication of the American Association for Pediatric Ophthalmology and Strabismus. 2002;6(1):9-14.

8. Carrigan, A., K., et al. Cost of Intraocular Lens versus Contact Lens Treatment after Unilateral Congenital Cataract Surgery: Retrospective Analysis at Age 1 Year. Ophthalmology Rochester 83 Hagerstown.2013.

9. Solebo AL, Russell-Eggitt I, Cumberland PM, Rahi JS. Risks and outcomes associated with primary intraocular lens implantation in children under 2years of age: the IoLunder2 cohort study. The British journal of ophthalmology. 2015:1471-1476.

10. Plager DA, Lynn MJ, Buckley EG, Wilson ME, Lambert SR. Complications, adverse events, and additional intraocular surgery 1 year after cataract surgery in the infant Aphakia Treatment Study. Ophthalmology. 2011;118(12):2330-2334. 
11. Lambert SR, Lynn MJ, Hartmann EE, et al. Comparison of contact lens and intraocular lens correction of monocular aphakia during infancy: a randomized clinical trial of HOTV optotype acuity at age 4.5 years and clinical findings at age 5 years. JAMA Ophthalmol.2014;132(6):676-682.

12. Gasper C, Trivedi RH, Wilson ME. Complications of Pediatric Cataract Surgery. Developments in ophthalmology. 2016;57:69-84.

13. Wilson ME. Intraocular lens implantation: has it become the standard of care for children? Ophthalmology. 1996;103(11):1719-1720.

14. Mandal AK, Netland PA. Glaucoma in aphakia and pseudophakia after congenital cataract surgery. Indian J Ophthalmol.2004;52(3):185-198.

15. Wood IC, Hodi S, Morgan L. Longitudinal change of refractive error in infants during the first year of life. Eye (London, England).1995;9 ( Pt 5):551-557.

16. G IG, M MY, N MB, D ME, As Eddin M. Incidence and Risk Factors of Early-onset Glaucoma following Pediatric Cataract Surgery in Egyptian Children: One-year Study. Journal of current glaucoma practice.2017;11(3):80-85.

17. Yi K, Chen TC. Aphakic glaucoma after congenital cataract surgery.International ophthalmology clinics. 2008;48(2):87-94.

18. Haargaard B, Ritz C, Oudin A, et al. Risk of glaucoma after pediatric cataract surgery. Investigative ophthalmology \& visual science. 2008;49(5):1791-1796.

19. Mataftsi A, Haidich AB, Kokkali S, et al. Postoperative glaucoma following infantile cataract surgery: an individual patient data meta-analysis. JAMA Ophthalmol. 2014;132(9):1059-1067.

20. Sahin A, Caca I, Cingu AK, et al. Secondary glaucoma after pediatric cataract surgery. International journal of ophthalmology.2013;6(2):216-220.

21. Freedman SF, Lynn MJ, Beck AD, Bothun ED, FH O, Lambert SR. Glaucoma-Related Adverse Events in the First 5 Years After Unilateral Cataract Removal in the Infant Aphakia Treatment Study. Jama Ophthalmology. 2015;133(8):907.

22. Vasavada AR, Vasavada V, Shah SK, et al. Five-Year Postoperative Outcomes of Bilateral Aphakia and Pseudophakia in Children up to 2 Years of Age: A Randomized Clinical Trial. American journal of ophthalmology. 2018;193:33-44.

23. Sefi-Yurdakul N, Berk AT. Primary and Secondary Intraocular Lens Implantations in Children With Pediatric Cataract: Visual Acuity and Strabismus at the Age of 2 Years and Older. Journal of pediatric ophthalmology and strabismus. 2017;54(2):97-102.

24. Lambert SR, Cotsonis G, DuBois L, et al. Comparison of the rate of refractive growth in aphakic eyes versus pseudophakic eyes in the Infant Aphakia Treatment Study. Journal of cataract and refractive surgery. 2016;42(12):1768-1773.

25. Hozo SP, Djulbegovic B, Hozo I. Estimating the mean and variance from the median, range, and the size of a sample. BMC medical research methodology. 2005;5:13.

26. RoB 2: a revised tool for assessing risk of bias in randomised trials. BMJ. 2019;366:-.

27. Wells G, Shea B, O'Connell D, et al. The Newcastle-Ottawa Scale (NOS) for Assessing the Quality of Non-Randomized Studies in Meta-Analysis. Vol 2000.

28. Ioannidis JPA, Patsopoulos NA, Evangelos E. Heterogeneity in meta-analyses of genome-wide association investigations. Plos One. 2006;2(9):e841. 
29. Higgins JPT, Thompson SG, Deeks JJ, Altman DG. Measuring inconsistency in meta-analyses. Bmj. 2003;327(7414):557-560.

30. Li Q, Fu T, Li ZE, et al. Optical correction of aphakia following unilateral infantile cataract removal. Acta ophthalmologica.2015;92(8):e657-e662.

31. Autrata R, Rehurek J, Vodickova K. Visual results after primary intraocular lens implantation or contact lens correction for aphakia in the first year of age. Ophthalmologica Journal international d'ophtalmologie International journal of ophthalmology Zeitschrift fur Augenheilkunde. 2005;219(2):72-79.

32. Trivedi RH, Wilson ME, Jr., Golub RL. Incidence and risk factors for glaucoma after pediatric cataract surgery with and without intraocular lens implantation. Journal of AAPOS : the official publication of the American Association for Pediatric Ophthalmology and Strabismus.2006;10(2):117-123.

33. Lambert SR, Lynn M, Drews-Botsch C, et al. A comparison of grating visual acuity, strabismus, and reoperation outcomes among children with aphakia and pseudophakia after unilateral cataract surgery during the first six months of life. Journal of AAPOS : the official publication of the American Association for Pediatric Ophthalmology and Strabismus. 2001;5(2):70-75.

34. Joshaghani M, Soleimani M, Foroutan A, Yaseri M. Visual Outcomes and Complications of Piggyback Intraocular Lens Implantation Compared to Aphakia for Infantile Cataract. Middle East African journal of ophthalmology. 2015;22(4):495-501.

35. Magli A, Forte R, Rombetto L. Long-term outcome of primary versus secondary intraocular lens implantation after simultaneous removal of bilateral congenital cataract. Graefe's archive for clinical and experimental ophthalmology $=$ Albrecht von Graefes Archiv fur klinische und experimentelle Ophthalmologie. 2013;251(1):309-314.

36. Lambert SR, Lynn M, Drews-Botsch C, et al. Optotype acuity and re-operation rate after unilateral cataract surgery during the first 6 months of life with or without IOL implantation. The British journal of ophthalmology. 2004;88(11):1387-1390.

37. Wong IB, Sukthankar VD, Cortina-Borja M, Nischal KK. Incidence of early-onset glaucoma after infant cataract extraction with and without intraocular lens implantation. The British journal of ophthalmology. 2009;93(9):1200-1203.

38. Felius J, Busettini C, Lynn MJ, Hartmann EE, Lambert SR. Nystagmus and related fixation instabilities following extraction of unilateral infantile cataract in the Infant Aphakia Treatment Study (IATS).Investigative ophthalmology \& visual science.2014;55(8):5332-5337.

39. Birch EE, Cheng C, Stager DR, Felius J. Visual Acuity Development After the Implantation of Unilateral Intraocular Lenses in Infants and Young Children. Journal of American Association for Pediatric Ophthalmology \& Strabismus. 2005;141(6):1173-1173.

40. Birch EE, Cheng C, Stager DR, Weakley DR, Sr. DRS. The critical period for surgical treatment of dense congenital bilateral cataracts. 2009;13(1):0-71.

41. Lambert SR, Buckley EG, Drews-Botsch C, Dubois L, Wilson ME. The infant aphakia treatment study: design and clinical measures at enrollment. Archives of Ophthalmology. 2010;128(1):21.

42. Lin DY, Wei LJ. The Robust Inference for the Cox Proportional Hazards Model. Journal of the American Statistical Association.1989;84(408):1074-1078.

43. Hatono N, Haruno I, Nishimoto H, Shirakawa S, Uga S, Ishikawa S. Histopathological study of anterior segments of the eye with the intraocular lens in the posterior chamber. Ophthalmologica Journal international d'ophtalmologie International journal of ophthalmology Zeitschrift fur Augenheilkunde. 1994;208(6):298-303.

44. Griener ED, Dahan E, Lambert SR. Effect of age at time of cataract surgery on subsequent axial length growth in infant eyes. Journal of cataract and refractive surgery. 1999;25(9):1209-1213. 
45. Michael I, Shmoish M, Walton DS, Levenberg S. Interactions between trabecular meshwork cells and lens epithelial cells: a possible mechanism in infantile aphakic glaucoma. Investigative ophthalmology $\& 3$ visual science. 2008;49(9):3981-3987.

46. Simon JW, Mehta N, Simmons ST, Catalano RA, Lininger LL. Glaucoma after pediatric lensectomy/vitrectomy. Ophthalmology.1991;98(5):670-674.

47. Brady KM, Atkinson CS, Kilty LA, Hiles DA. Cataract surgery and intraocular lens implantation in children. American journal of ophthalmology. 1995;120(1):1-9.

48. Hiles DA. Visual rehabilitation of aphakic children. III. Intraocular lenses. Survey of ophthalmology. 1990;34(5):371-379.

49. Greenwald MJ, Glaser SR. Visual outcomes after surgery for unilateral cataract in children more than two years old: posterior chamber intraocular lens implantation versus contact lens correction of aphakia. Journal of AAPOS : the official publication of the American Association for Pediatric Ophthalmology and Strabismus.1998;2(3):168-176.

50. Shrestha UD, Shrestha MK. Visual Axis Opacification in Children Following Paediatric Cataract Surgery. JNMA; journal of the Nepal Medical Association. 2014;52(196):1024-1030.

51. Knight-Nanan D, O'Keefe M, Bowell R. Outcome and complications of intraocular lenses in children with cataract. Journal of cataract and refractive surgery. 1996;22(6):730-736.

52. Lundvall A, Zetterstrom C. Complications after early surgery for congenital cataracts. Acta ophthalmologica Scandinavica.1999;77(6):677-680.

53. Wilson ME, Jr., Trivedi RH, Buckley EG, et al. ASCRS white paper. Hydrophobic acrylic intraocular lenses in children. Journal of cataract and refractive surgery. 2007;33(11):1966-1973.

54. Kirwan C, Lanigan B, O'Keefe M. Glaucoma in aphakic and pseudophakic eyes following surgery for congenital cataract in the first year of life. Acta ophthalmologica. 2010;88(1):53-59.

55. Trivedi RH, Wilson ME, Jr., Bartholomew LR, Lal G, Peterseim MM. Opacification of the visual axis after cataract surgery and single acrylic intraocular lens implantation in the first year of life.Journal of AAPOS : the official publication of the American Association for Pediatric Ophthalmology and Strabismus.2004;8(2):156-164.

Table 1. Characteristics of the included studies $(n=10)$.

\begin{tabular}{lllllll}
\hline Study & Year & Designs & Primary IOL & Primary IOL & Primary IOL & Primary aphakia \\
\hline & & & N(eye) & Age at Surgery (months) & Age at last visit (years) & N(eye) \\
IATS group & 2014 & RCT & 57 & 1.8 & 4.5 & 57 \\
Vasavada A R & 2018 & RCT & 29 & 6.3 & $i 5$ & 25 \\
Felius J & 2014 & RCT & 57 & 1.8 & 4.5 & 57 \\
Lambert SR & 2001 & RS & 12 & 2.8 & 1.5 & 13 \\
Trivedi R H & 2006 & RS & 41 & 1.9 & $i 1$ & 42 \\
Magli A & 2013 & RS & 30 & 7.2 & 9.5 & 36 \\
Lambert SR & 2004 & RS & 12 & 2.8 & 4.3 & 13 \\
Autrata R & 2005 & RS & 18 & 3.4 & 4.9 & 23 \\
Joshaghani M & 2015 & RS & 23 & 7.5 & 7.0 & 32 \\
Wong B & 2009 & RS & 37 & 4.3 & $i 3$ & 61 \\
Qian L & 2014 & RCT & 30 & $i 24$ & $i 3$ & 30 \\
\hline
\end{tabular}




\begin{tabular}{llll}
\hline Indicators & $\mathbf{t}$ & $\mathbf{d f}$ & $\mathbf{p}$ \\
\hline VA & 1.904 & 5 & 0.115 \\
Glaucoma & 1.075 & 5 & 0.331 \\
Strabismus & -0.345 & 4 & 0.748 \\
Nystagmus & -0.832 & 2 & 0.493 \\
Retinal detachment & -0.438 & 1 & 0.737 \\
Glaucoma surgery & 87.023 & 1 & 0.007 \\
Strabismus surgery & -0.879 & 2 & 0.472 \\
VAO clearing & -2.586 & 3 & 0.081 \\
\hline
\end{tabular}

Table 2. Egger's test for each outcomes

\section{Figure legend}

Figure 1. Flow chart of study selection

Figure 2. Risk of bias graph of RCT studies.

Figure 3. Risk of bias graph of retrospective studies.

Figure 4. Forest plot of VA.

Figure 5. Forest plot of glaucoma.

Figure 6. Forest plot of strabismus after subgroup analysis.

Figure 7. Forest plot of nystagmus.

Figure 8. Forest plot of retinal detachment.

Figure 9. Forest plot of VAO clearing. 


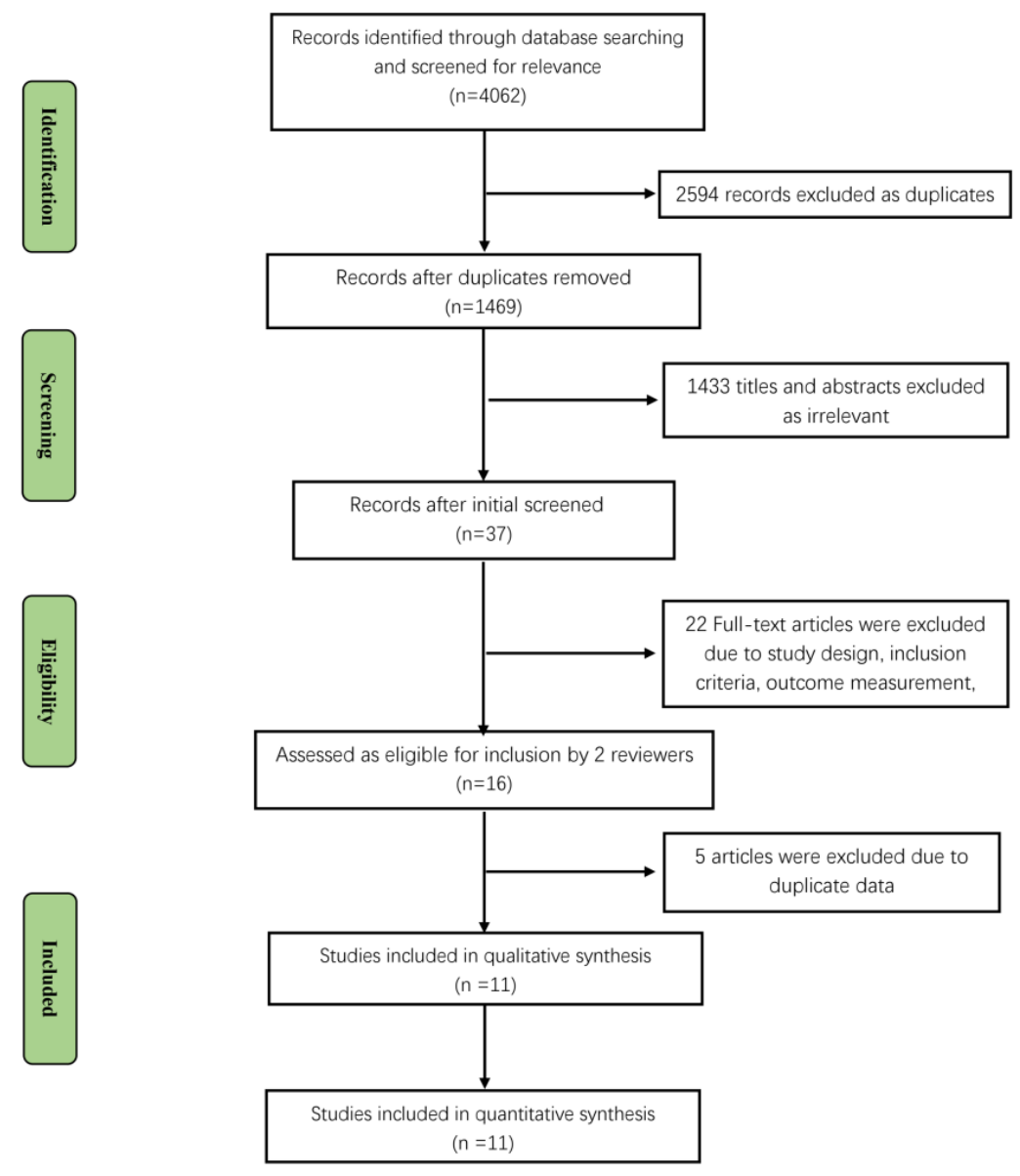

Figure 1. Flow chart of study selection 

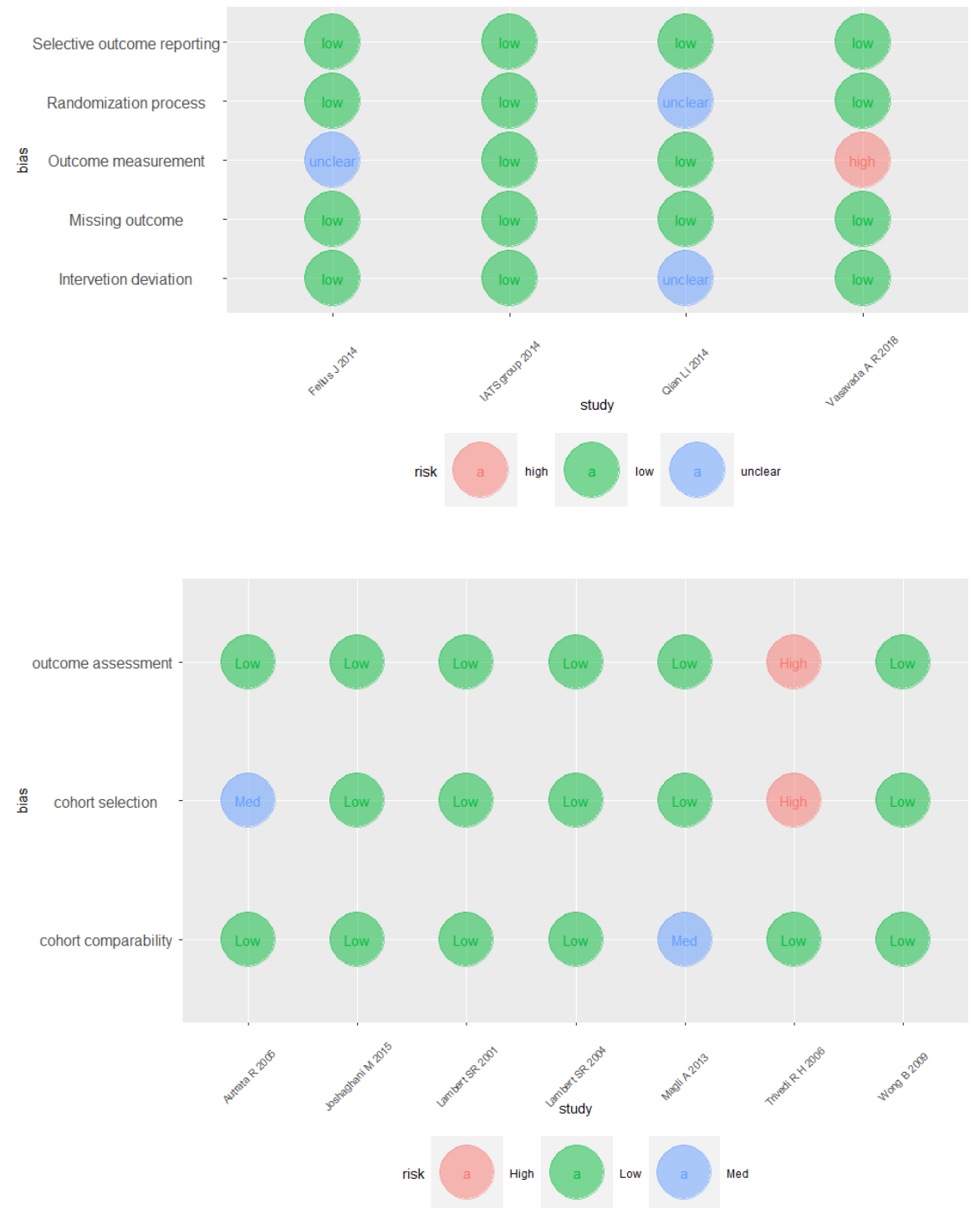

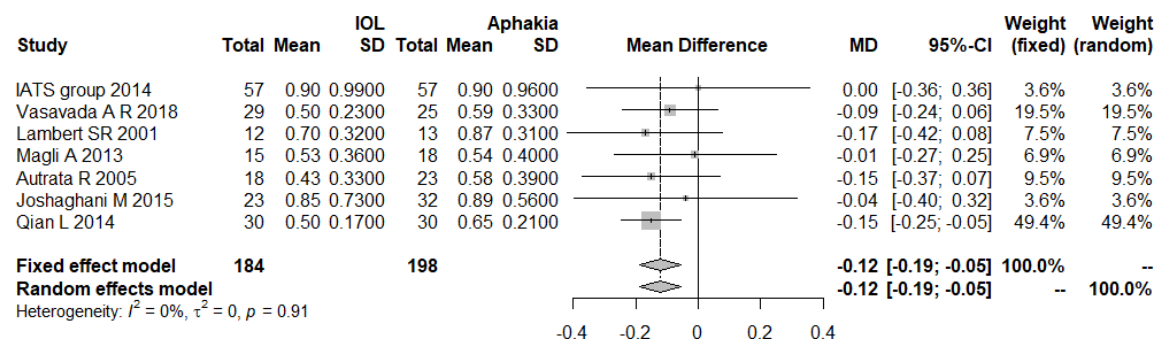




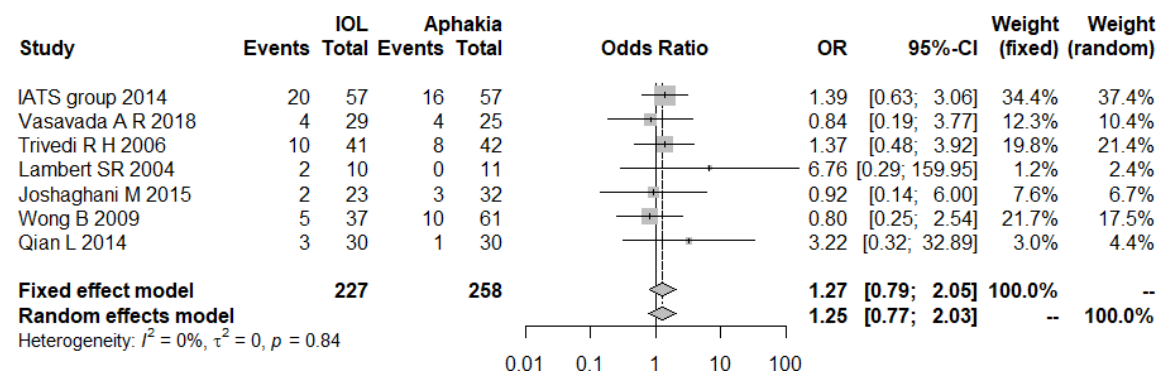

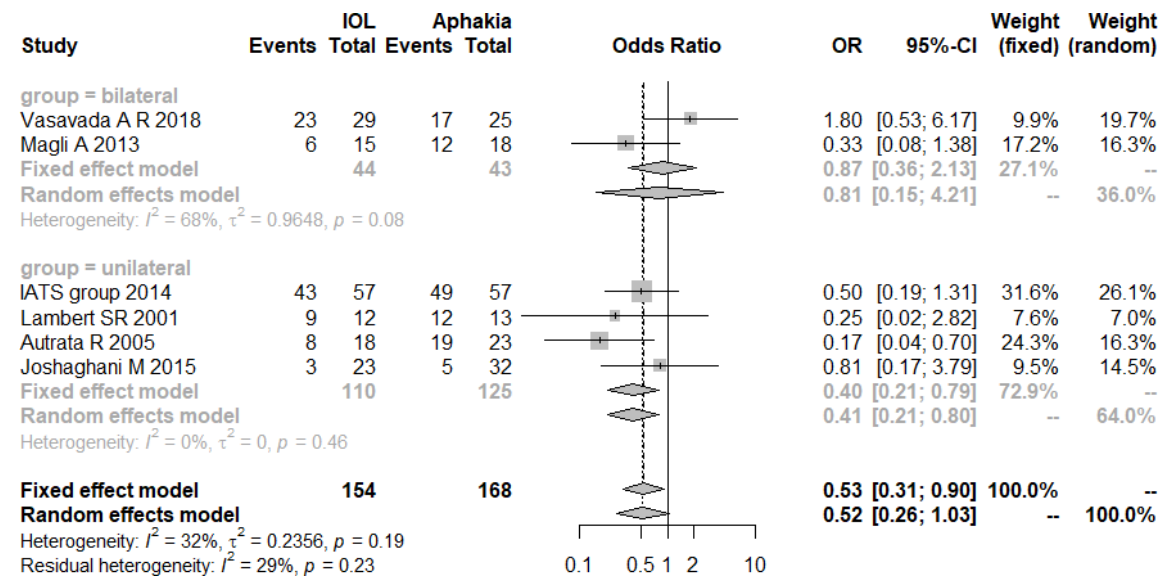

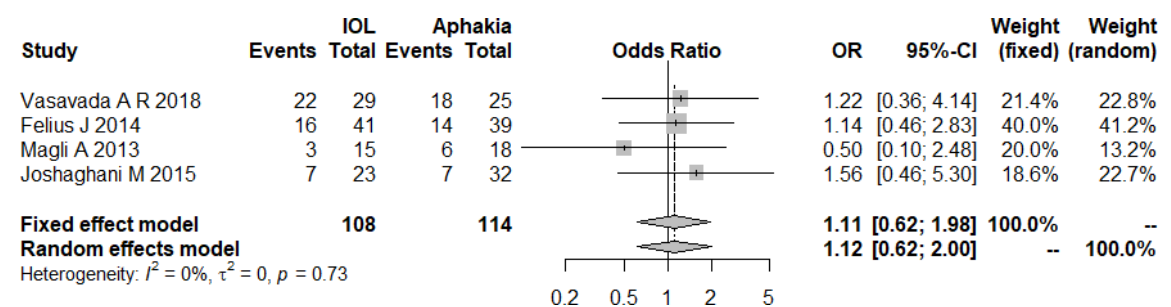

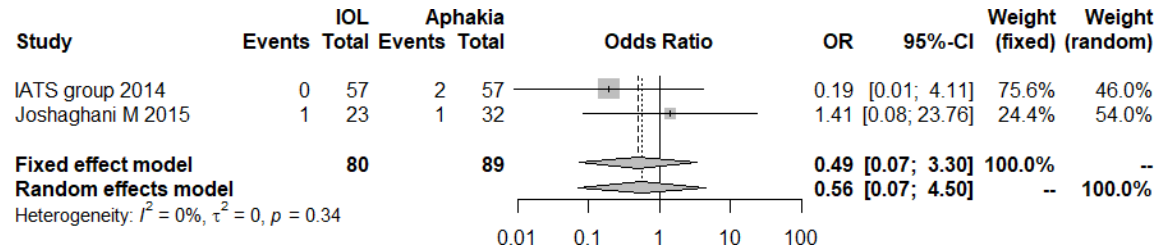

\begin{tabular}{lrrrr} 
Study & \multicolumn{2}{c}{$\begin{array}{c}\text { primary } \\
\text { Events }\end{array}$} & $\begin{array}{r}\text { Total Evendary } \\
\text { sents }\end{array}$ & Total \\
IATS group 2014 & 39 & 57 & 8 & 57 \\
Vasavada A R 2018 & 3 & 29 & 2 & 25 \\
Joshaghani M 2015 & 8 & 23 & 2 & 32 \\
Wong B 2009 & 15 & 37 & 4 & 61 \\
Qian L 2014 & 16 & 30 & 2 & 30 \\
& & & & \\
Fixed effect model & & $\mathbf{1 7 6}$ & & $\mathbf{2 0 5}$ \\
Random effects model & & & \\
Heterogeneity: $I^{2}=23 \%, \tau^{2}=0.1446, p=0.27$ & &
\end{tabular}

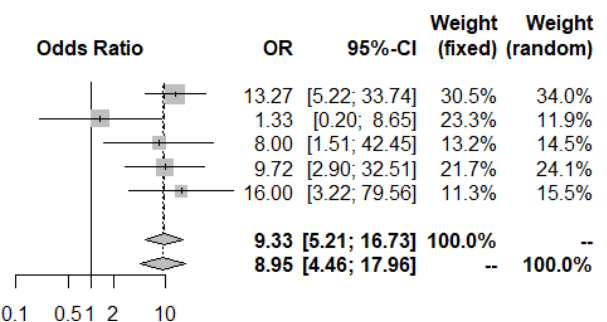

